\title{
O TRABALHO DO FISIOTERAPEUTA NOS NÚCLEOS AMPLIADOS DE SAÚDE DA FAMÍLIA E ATENÇÃO BÁSICA (NASF-AB) EM REGIÃO METROPOLITANA
}

\author{
THE WORK OF PHYSIOTHERAPY IN THE EXPANDED \\ FAMILY HEALTH AND PRIMARY CARE CENTERS (NASF- \\ AB) IN A METROPOLITAN REGION
}

Marcia Maristela S. da S. Lima (ORCID: 0000-0001-5321-764X.)

Larissa S. Santana (ORCID: 0000-0001-9664-043X.) ${ }^{1}$

Ítalo Ricardo Santos Aleluia (ORCID: 0000-0001-9499-6360.) ${ }^{2}$

Fabiane Costa Santos Fontoura (ORCID: 0000-0003-0537-7512.)

Elzo Pereira Pinto Júnior (ORCID: 0000-0002-6977-2071.) ${ }^{4}$

Autor Correspondente Ítalo Ricardo Santos Aleluia

E-mail: italoaleluia@yahoo.com.br

\begin{abstract}
RESUMO
Compostos por equipes multiprofissionais, incluindo a participação de fisioterapeutas, os Núcleos de Apoio à Saúde da Família (Nasf-AB) foram criados para ampliar o escopo das ações de Atenção Primária à Saúde e apoiar a inserção da Estratégia Saúde da Família na rede de serviços de saúde. Tendo isso em vista, o objetivo deste estudo foi analisar o processo de trabalho do fisioterapeuta nos Nasf-AB, em quatro sistemas municipais de saúde da Região Metropolitana (RM) de Salvador, na Bahia. Para tanto, realizou-se um estudo de múltiplos casos sob abordagem qualiquantitativa, a partir de entrevistas semiestruturadas, investigando os seguintes aspectos: infraestrutura das unidades; formação profissional; relação com equipe multiprofissional e comunidade; desafios e perspectivas da gestão municipal e gerência das unidades. Os dados foram processados no software Nvivo, versão 10.0, e submetidos a uma análise de conteúdo. Os principais dificultadores relacionaram-se à formação acadêmica do fisioterapeuta centrada na reabilitação, à falta de material para assistência e de transporte para locomoção na área de abrangência e ao pouco conhecimento sobre as atribuições do fisioterapeuta por parte da equipe. A boa relação com a gerência da unidade e com a comunidade foram facilidades destacadas. A oferta de cursos de capacitação, melhores condições para locomoção dos profissionais, bem como a definição das atribuições dos profissionais, parecem ser imprescindíveis para um processo de trabalho mais eficaz. O maior desafio pautou-se na mudança do modelo de atenção, a fim de centrar as práticas de saúde do fisioterapeuta na prevenção e na promoção da saúde.
\end{abstract}

Palavras-chave: Fisioterapia; Atenção Primária à Saúde; Saúde da Família; Sistema Único de Saúde.

\begin{abstract}
Composed of multiprofessional teams, including the participation of physiotherapists, the Family Health Support Centers (Nasf-AB) were created to expand the scope of Primary Health Care actions and support the insertion of the Family Health Strategy in the health network of health services. With this in mind, the objective of this study was to analyze the physiotherapist's work process in Nasf-AB, in four municipal health systems in the Metropolitan Region (RM) of Salvador, Bahia. For this purpose, a study of multiple cases was carried out under a qualitative and quantitative approach, based on semi-structured interviews, investigating the following aspects: infrastructure of the units; professional qualification; relationship with the multiprofessional team and the community; challenges and perspectives of municipal management and unit management. The data were processed in the Nvivo software, version 10.0, and submitted to a content analysis. The main difficulties were related to the academic training of the physiotherapist centered on rehabilitation, the lack of material for assistance and transportation for locomotion in the area covered, and the little knowledge about the physiotherapist's duties on the part of the team. The good relationship with the unit's management and with the community were highlights. The provision of training courses, better conditions for locomotion of professionals, as well as the definition of the professionals' duties seem to be essential for a more effective work process. The biggest challenge was based on changing the model of care, in order to focus the physiotherapist's health practices on prevention and health promotion.
\end{abstract}

${ }^{1}$ Curso de Fisioterapia, Centro Universitário UniFTC.

${ }^{2}$ Centro das Ciências Biológicas e da Saúde, Universidade Federal do Oeste da Bahia.

Hospital Especializado Otávio Mangabeira, Secretaria de Saúde do Estado da Bahia.

${ }^{4}$ Centro de Integração de Dados e Conhecimentos para a Saúde, Instituto Gonçalo Moniz, Fiocruz-Bahia. 


\section{INTRODUÇÃO}

A Atenção Primária à Saúde (APS) no Brasil tem sido alvo de pesquisas avaliativas e prioridades de políticas públicas no que diz respeito à força de trabalho, ao financiamento, à capacitação dos profissionais e à avaliação das práticas de saúde e de gestão1. No País, as reformas do Sistema Único de Saúde (SUS) culminaram na implantação e na expansão de diversos serviços voltados aos cuidados primários, sobretudo na década de 1990, com a criação do Programa dos Agentes Comunitários de Saúde (Pacs) e do Programa Saúde da Família (PSF) ${ }^{1}$.

A regulamentação da Política Nacional de Atenção Básica (PNAB) adotou a Estratégia Saúde da Família (ESF) como modelo prioritário de reorganização do SUS. Esse modelo é centrado nos seguintes atributos: ordenamento, integração, coordenação, comunicação das ações e serviços de saúde e primeiro contato com a população ${ }^{1-3}$.

Apesar dos avanços da PNAB voltados ao sistema de saúde brasileiro, a previsão de composição das equipes de saúde demonstrou que algumas categorias não foram contempladas inicialmente, inclusive a de fisioterapeutas. Contudo, o amplo espectro de ações e responsabilidades inerentes às Equipes de Saúde da Família (EqSF) deflagrou a necessidade de articular outros núcleos de saberes que pudessem colaborar para dar maior concretude às ações previstas na política, sendo um dos fatores determinantes para a criação dos Núcleos Ampliados de Saúde da Família e Atenção Básica (Nasf-AB) em 2008 4,5 .

Os Nasf-AB correspondem a equipes constituídas por profissionais de diferentes ocupações não coincidentes com objetivo de ampliar a abrangência, o escopo e a resolubilidade das ações de APS. No processo de trabalho dos Nasf$-\mathrm{AB}$, as diretrizes normativas propõem a importância de ações orientadas pelo apoio e complementariedade do trabalho, a partir das demandas identificadas em conjunto com as EqSF, para instituir a plena integralidade do cuidado biopsicossocial aos usuários ${ }^{4}$. Nesse cenário, houve ampliação da composição das equipes de cuidados primários, a exemplo de recomendações para inserção dos profissionais de fisioterapia ${ }^{4,5}$.

A possibilidade de inclusão do fisioterapeuta nos Nasf-AB não necessariamente se refletiu na suficiência de garantia político-institucional para sua inserção em sistemas municipais de saúde e para o aumento da cobertura desses profissionais por habitantes na APS. A vinculação das contratações, sob responsabilidade política dos gestores municipais, tornou-se um importante desafio que transcorre a gestão do trabalho e fortalece contradições gerenciais e assistenciais que não dialogam com as necessidades de saúde da população, tampouco com as práticas de saúde dos fisioterapeutas, necessárias à garantia de resolubilidade das ações a serem desenvolvidas no âmbito dos Nasf-AB ${ }^{6}$.

O discurso nos documentos que normatizam as atribuições do fisioterapeuta no Nasf-AB denota concepções que remetem direcionalidade oposta ao cuidado integral, por exemplo, quando descreve práticas restritas ao componente da reabilitação física, secundarizando múltiplas potencialidades de atuação desse profissional na prevenção e na promoção da saúde ${ }^{7-9}$.

A reatualização da $\mathrm{PNAB}$, em 2011, reorganizou as tipologias de Nasf-AB e redefiniu o número de equipe apoiadas ${ }^{10}$. Isso criou outros desafios relativos ao aumento do número de equipes apoiadas e aos desequilíbrios importantes na relação oferta-demanda das práticas de saúde dos fisioterapeutas, principalmente porque a carga horária de trabalho dos profissionais de fisioterapia, estabelecida em lei federal, é inferior à da maioria dos profissionais que podem compor os Nasf-AB ${ }^{5-8}$. 
Diversos estudos apontaram importantes entraves relativos à formação profissional do fisioterapeuta, visto que, em grande parte, essa formação encontra-se centrada no modelo de atenção biomédico e distanciada das práticas de saúde necessárias ao processo de trabalho no âmbito da APS $^{5-9}$.

Pesquisas em diferentes municípios brasileiros têm identificado uma série de fatores restritivos ao trabalho do fisioterapeuta no Nasf-AB que varia da precária infraestrutura física das unidades de saúde para a escassez de recursos materiais à baixa cobertura de profissionais da área para dar maior concretude às ações de retaguarda assistencial para $\mathrm{EqSF}^{7-12}$.

Outros estudos evidenciaram aspectos político-institucionais, decorrentes de desconhecimentos dos usuários, profissionais e gestores, sobre as potencialidades do fisioterapeuta na APS, além do curto tempo disponível para esses profissionais realizarem atendimentos individuais e coletivos e, principalmente, em razão de sua inserção recente no âmbito dos cuidados primários8,9. Em outros cenários, as práticas de saúde da fisioterapia ocorrem desarticuladas das EqSF, priorizando a atenção individual em detrimento de ações coletivas. Além disso, as estratégias dos profissionais são insuficientes para realização das atividades integradas com a $\mathrm{ESF}^{13,14}$.

Vale reiterar, ainda, a incipiência do próprio Conselho Federal de Fisioterapia e Terapia Ocupacional (Coffito) com relação ao reconhecimento da Saúde Coletiva como um dos campos de atuação do fisioterapeuta, uma vez que essa regulamentação ocorreu somente um ano após a criação dos Nasf-AB, em $2009^{11}$.

Na literatura, não foram evidenciados recortes investigativos sobre o processo de trabalho do fisioterapeuta nos Nasf-AB, considerando, simultaneamente, os determinantes locais das equipes de APS e os da gestão municipal. As pesquisas também têm sido restritas a estudos de caso único municipais, com ausência de investigações em múltiplos contextos, inclusive singulares, como as Regiões Metropolitanas (RM), que se caracterizam pela importância demográfica, pela concentração populacional e pela complexidade territorial ${ }^{15}$.

Considerando o exposto, ainda há lacunas importantes sobre a organização das práticas e dos processos de trabalho da fisioterapia no âmbito do Nasf-AB, em sistemas municipais de RM. Dessa forma, o objetivo deste estudo foi analisar o processo de trabalho do fisioterapeuta nos Nasf-AB, em quatro sistemas municipais de saúde da RM de Salvador, na Bahia.

\section{MÉTODOS}

Trata-se de um estudo de caso regional, em quatro sistemas municipais de saúde da RM de Salvador, na Bahia. De abordagem qualiquantitativa, esta pesquisa realizou dois níveis de análise: o âmbito local das equipes Nasf-AB e a gestão municipal.

Para a realização da pesquisa, todos os participantes assinaram o Termo de Consentimento Livre e Esclarecido (TCLE). Além disso, obteve-se a anuência institucional na Secretaria da Saúde do Estado da Bahia (Sesab) e municípios individualmente. A pesquisa foi aprovada pelo Comitê de Ética em Pesquisa da Sesab, sob parecer $n^{\circ} 618.875 / 2013$.

Inicialmente, foram selecionados os municípios elegíveis para a pesquisa e, posteriormente, identificados os sujeitos do estudo. Na seleção, levou-se em consideração a consulta ao Plano Diretor de Regionalização da Bahia, com o objetivo de identificar os municípios que compõem a RM, elegendo, por conveniência, aqueles que atendiam aos seguintes critérios: possuir Nasf-AB implantado há, pelo menos, um ano e possuir fisioterapeuta atuante no Nasf-AB. Essas informações foram levantadas no Cadastro Nacional dos Estabelecimentos de Saúde (CNES) e validadas diretamente com as secretarias municipais de saúde. Por fim, quatro sistemas municipais de saúde foram selecionados para o estudo.

Após seleção dos municípios, realizou-se o levantamento das informações sobre as equipes NASF-AB. Nessa 
etapa, consideraram-se as equipes com fisioterapeutas atuantes que apoiavam, pelo menos, de três a nove EqSF, considerando as atualizações das modalidades de equipes previstas na nova PNAB. Em seguida, foram selecionados fisioterapeutas atuantes no Nasf-AB há, no mínimo, seis meses, sob vínculo com a secretaria municipal ou estadual de saúde, e que aceitaram assinar o TCLE. Foram pré-selecionados dez profissionais, sendo dois excluídos por tempo de atuação no Nasf-AB inferior ao adotado nos critérios de inclusão, restando, portanto, oito entrevistados.

A produção dos dados ocorreu por meio de entrevistas gravadas na íntegra, com auxílio de gravador de áudio digital, utilizando um roteiro semiestruturado, elaborado pela equipe de pesquisa. $\mathrm{O}$ roteiro foi constituído das seguintes assertivas e questões subjetivas: grau de concordância e discordância, entre os entrevistados, sobre elementos relativos à formação acadêmica (graduação e pós-graduação); suficiência da formação para atender às necessidades de trabalho no Nasf-AB; oferta de educação permanente pelas secretarias municipais de saúde e voltadas para o Nasf-AB; problemas relacionados com materiais/ insumos; relação fisioterapeuta-comunidade; relação fisioterapeuta com equipe Nasf-AB e EqSF apoiadas; relação com a gestão municipal e gerência das unidades; desafios e perspectivas. Essa categorização de elementos do instrumento utilizou como base a revisão de literatura realizada ${ }^{4-9}$. O instrumento do estudo foi submetido a um pré-teste com três profissionais de municípios localizados no recôncavo da Bahia.

O grau de concordância foi mensurado por intermédio de escala Likert ${ }^{15}$, variando de "nunca", "raras vezes", "algumas vezes", "na maioria das vezes" a "sempre". Já nas questões subjetivas, foram aprofundadas informações qualitativas sobre as dificuldades e facilidades que transcorriam o processo de trabalho nos diferentes elementos citados anteriormente.

Além disso, consideraram-se as seguintes informações para caracterização dos entrevistados: idade, sexo, tempo de formação, tempo de atuação no SUS, tempo de atuação na APS, tempo atuação na equipe e formações complementares. Os pesquisadores também utilizaram o registro em diário de campo, sob observações e impressões.

As variáveis quantitativas foram processadas no Microsoft Word Excel ${ }^{\circledR}$ 2017, construindo uma análise segundo estatística descritiva, com as frequências absolutas e relativas, das variáveis de caracterização dos participantes e das respostas, presentes na gradação da escala Likert.

As entrevistas foram transcritas na íntegra com uso do Microsoft Word $\AA 2017$ e, em seguida, exportadas e processadas no Software QSR NVIVO ${ }^{\circledR}$, versão 10.0, para análise qualitativa. Para esta análise, foi realizada a leitura em profundidade do material, pré-análise para tabulação dos achados e codificação do material empírico, por núcleo de sentido, organizado nas seguintes categorias: 1) infraestrutura; 2) relação com a equipe multiprofissional; 3) formação profissional; 4) relação com a comunidade; 5) relação com a gestão municipal; 6) desafios e perspectivas da atuação. Em seguida, foi construído um resumo narrativo com interpretação dos resultados, argumentos e evidências, organizados em uma matriz de análise (quadro 1) por meio do cotejamento dos excertos dos entrevistados, comparando-se os municípios para identificar semelhanças e divergências entre os fatores contextuais e o processo de trabalho dos fisioterapeutas nos cenários pesquisados.

Quadro 1. Matriz para a análise dos dados das entrevistas

\begin{tabular}{|c|l|l|l|l|l|l|l|l|}
\hline $\begin{array}{c}\text { Categorias } \\
\text { empíricas }\end{array}$ & Município & E1 & E2 & E3 & E4 & E4 & E5 & $\begin{array}{c}\text { Síntese } \\
\text { Horizontal }\end{array}$ \\
\hline & & & & & & & & \\
\hline & & & & & & & & \\
\hline Síntese vertical & & & & & & & & \\
\hline
\end{tabular}

Fonte: elaboração própria.

$\mathrm{E}=$ entrevistado. 


\section{RESULTADOS}

Os resultados evidenciaram que $75 \%$ dos entrevistados eram do sexo feminino, com variações de idade de 25 a 55 anos, tendo a maioria (80\%) menos de 10 anos de formação. A atuação dos fisioterapeutas na APS variou de 6 meses a 15 anos, com média de 5 anos. No Nasf-AB, a maioria (70\%) dos entrevistados tinha menos de 4 anos de atuação. Esses achados ratificam a incipiência de inserção dos profissionais no âmbito da APS.

Sobre a formação acadêmica, $62,5 \%$ dos entrevistados consideraram que foi suficiente para o trabalho no Nasf-AB. Contudo, identificou-se que as vivências de formação profissional dos fisioterapeutas na graduação possuíram raros contatos com os serviços de APS e contextos comunitários, revelando pouca trajetória com práticas de prevenção e promoção da saúde. Isso foi mais predominante em trajetórias acadêmicas de profissionais com tempo de formação superior à implantação dos Nasf-AB.

A região estudada apresenta alta complexidade política, em função dos sistemas municipais estarem incorporados a mais de uma região de saúde (a região de Camaçari e de Salvador, ambas na Bahia), de acordo com o Plano Diretor de Regionalização do estado da Bahia. O município de Salvador concentra os serviços de média e alta complexidade, sendo referência para toda região com, respectivamente, $50 \%$ e 93\% da produção ambulatorial, conforme diagnóstico apresentado no Plano Estadual de Saúde 2012-2015 ${ }^{16}$.
Na RM de Salvador, Bahia, residem aproximadamente 3.445.693 habitantes, distribuídos em 10 municípios: Camaçari, Dias D’ávila, Simões Filho, Candeias, Itaparica, Lauro de Freitas, Madre de Deus, Salvador, Vera Cruz e Salinas da Margarida. De acordo com o Caderno de Monitoramento e Avaliação da Atenção Básica (Camab), do estado da Bahia, em dezembro de 2019, a maioria dos municípios possuía cobertura de APS estimada acima de $70 \%$, com exceção dos municípios de Simões Filho (44,13\%), Salvador (26,08\%), Camaçari $(57,55 \%)$ e Candeias (63,68\%). Até outubro de 2019 , existiam 24 equipes de Nasf-AB implantadas na RM de Salvador, sendo todos na modalidade tipo $1^{17}$.

Não existiam ações de Educação Permanente em Saúde (EPS) para os profissionais do Nasf-AB, inclusive fisioterapeutas, em todos os cenários. As ações de qualificação eram restritas aos treinamentos pontuais que não possuíam características e intencionalidades voltadas para o processo de trabalho em saúde. Isso foi ratificado por todos os entrevistados, que afirmaram raras ocorrências de cursos preparatórios, fóruns ou reuniões semanais, apenas de caráter administrativo.

Em todas as categorias de análise, houve discordância entre os profissionais com relação à existência de problemas no processo de trabalho nos municípios estudados. Os contextos pesquisados possuíam maior frequência de fatores restritivos, relacionados com a infraestrutura, e menor na relação dos fisioterapeutas com a gerência das EqSF. A compilação das respostas da escala Likert estão sumarizadas no quadro 2 . 
Quadro 2. Presença de dificuldades no processo de trabalho dos fisioterapeutas atuantes nos Nasf-AB da RM de Salvador, Bahia, segundo categorias de análise e município

\begin{tabular}{|c|c|c|c|c|c|}
\hline Dimensão & Nunca & $\begin{array}{l}\text { Raras } \\
\text { vezes }\end{array}$ & $\begin{array}{l}\text { Algumas } \\
\text { vezes }\end{array}$ & $\begin{array}{l}\mathrm{Na} \\
\text { maioria } \\
\text { das } \\
\text { vezes }\end{array}$ & $\begin{array}{l}\text { Sempr } \\
\text { e }\end{array}$ \\
\hline \multicolumn{6}{|c|}{ Infraestrutura } \\
\hline $\mathrm{Ma}$ & & & $\mathrm{X}$ & & $\mathrm{X}$ \\
\hline $\mathrm{Mb}$ & & & $X$ & & \\
\hline Mc & & $\mathrm{X}$ & $\mathrm{X}$ & $\mathrm{X}$ & \\
\hline Md & & $\mathrm{X}$ & $\mathrm{X}$ & & \\
\hline \multicolumn{6}{|c|}{ Relação com a comunidade } \\
\hline $\mathrm{Ma}$ & $X X$ & & & & \\
\hline $\mathrm{Mb}$ & & & $\mathrm{X}$ & & \\
\hline Mc & $\mathrm{X}$ & & $\mathrm{XX}$ & & \\
\hline Md & & $\mathrm{X}$ & $\mathrm{X}$ & & \\
\hline \multicolumn{6}{|c|}{$\begin{array}{l}\text { Relação com a equipe } \\
\text { multidisciplinar }\end{array}$} \\
\hline $\mathrm{Ma}$ & $\mathrm{XX}$ & & & & \\
\hline $\mathrm{Mb}$ & $\mathrm{X}$ & & & & \\
\hline Mc & $\mathrm{XXX}$ & & & & \\
\hline Md & & & $\mathrm{XX}$ & & \\
\hline \multicolumn{6}{|c|}{ Relação com a gestão } \\
\hline $\mathrm{Ma}$ & $X$ & & & $\mathrm{X}$ & \\
\hline $\mathrm{Mb}$ & $\mathrm{X}$ & & & & \\
\hline Mc & $\mathrm{XX}$ & & $\mathrm{X}$ & & \\
\hline Md & $X X$ & & & & \\
\hline \multicolumn{6}{|c|}{ Relação com a gerência } \\
\hline $\mathrm{Ma}$ & $\mathrm{X}$ & & $\mathrm{X}$ & & \\
\hline $\mathrm{Mb}$ & $\mathrm{X}$ & & & & \\
\hline Mc & $X X X$ & & & & \\
\hline $\mathrm{Md}$ & $X X$ & & & & \\
\hline
\end{tabular}

Fonte: elaboração própria.

Legenda: Ma = Município "a"; Mb = Município "b"; Mc = Município "c"; Md = Município "d"; "X" = fisioterapeuta entrevistado. 
$\mathrm{Na}$ "infraestrutura", havia problemas de deslocamento das equipes Nasf-AB e insuficiência de recursos materiais de trabalho, sobretudo para realização de ações de educação em saúde. A logística complexa do atendimento domiciliar devido às barreiras geográficas, bem como a falta de estrutura física nas unidades de saúde para a guarda de materiais, insumos, documentos e relatórios, determinava ações improvisadas e inviabilizavam o monitoramento do trabalho dos fisioterapeutas pela gestão municipal e gestão do Nasf-AB. Metade dos profissionais indicou dificuldade de integrar suas ações com a comunidade. Ainda que houvesse alguma adesão às ações da fisioterapia, era comum demanda por atendimentos individuais de reabilitação. Um dos pontos de potencialidades nos cenários pesquisados foi a boa relação dos fisioterapeutas com os demais profissionais do Nasf-AB e EqSF apoiadas, ainda que ocorressem alguns conflitos comunicacionais, decorrentes do desconhecimento das atribuições do fisioterapeuta na APS. Esse fato faz alusão a uma fragilidade histórica no modelo de formação em saúde, no qual os profissionais aprendem separados para atuarem juntos no contexto dos serviços de saúde. Existia boa adesão dos profissionais das EqSF apoiadas às ações propostas pelos fisioterapeutas, quando comparado às equipes que eram de modalidade tradicional (sem o modelo de Saúde da Família).

Em todos os municípios, havia desigualdade na relação entre o número de equipes Nasf-AB implantadas e o número de EqSF apoiadas. Alguns Nasf-AB apoiavam até dez equipes, contrapondo-se ao limite máximo estabelecido nas diretrizes da PNAB (até nove), tendo em vista que todas as equipes estudadas eram da modalidade Nasf-AB, do tipo 1 . Isso saturava o processo de trabalho dos fisioterapeutas, em decorrência da alta demanda por atendimentos individuais de reabilitação, colaborando para a reprodução de práticas de saúde em consonância com o modelo de atenção curativista e com a baixa resolutividade das demandas coletivas da comunidade. Uma potencialidade foi o bom relacionamento com gerentes das EqSF e gestores do Nasf-AB. Havia dificuldade de comunicação dos profissionais com os secretários municipais de saúde e coordenações de APS, evidenciada pelo longo tempo de resposta da gestão às necessidades e demandas levantadas pelos fisioterapeutas em relação à infraestrutura, aos recursos materiais e aos insumos.

A relação interprofissional entre os fisioterapeutas e as gerências das EqSF se mostrou um ponto de potencialidade para o processo de trabalho em comparação dessa relação com a gestão municipal e a coordenação da APS. Em um dos municípios, a gestão do Nasf-AB é exercida por fisioterapeuta que também atuava na assistência do próprio Núcleo. O único sistema municipal no qual se evidenciou entraves na relação com os gerentes de APS apresentava fragilidades decorrentes da falta de pessoal para monitorar e coordenar as ações do Nasf-AB.

Ainda sobre os elementos anteriores, as impressões registradas em diário de campo coadunam com a possibilidade de alguns dos entrevistados não terem revelado, de fato, problemáticas relacionais aos gerentes de APS, em virtude de constrangimentos ocasionados pela precarização dos vínculos trabalhistas, o que pode explicar, em sua maioria, resultados tão positivos.

Sobre as perspectivas para o trabalho no Nasf-AB, houve unanimidade dos profissionais sobre a necessidade de maior integração da equipe multiprofissional para o desempenho de ações educativas em saúde de forma regular, a fim de impactar na diminuição de internações e agravos.

Por fim, destacam-se, como limitações operacionais deste estudo: as dificuldades na obtenção da anuência institucional em alguns municípios da RM, o que resultou na exclusão de alguns deles; a indisponibilidade dos profissionais para realização das entrevistas, pela intensa agenda de trabalho dos fisioterapeutas na região; a disponibilidade dos pesquisadores para deslocamento da capital baiana até os municípios pesquisados. 


\section{DISCUSSÃO}

A formação acadêmica do fisioterapeuta deve estar centrada nas Diretrizes Curriculares Nacionais, que objetivam dotar os profissionais de conhecimentos necessários para o exercício das competências e habilidades inerentes ao SUS e à APS18. Historicamente, o fisioterapeuta ainda é visto por gestores, profissionais e população como um agente de práticas centradas na reabilitação, como ficou evidente nesta pesquisa. Outros estudos ressaltam que, quando inseridos na APS, os profissionais de fisioterapia podem desempenhar importante papel na realização de ações de promoção, prevenção e educação em saúde ${ }^{11-14,19}$.

Houve unanimidade dos profissionais sobre a priorização da formação acadêmica à reabilitação, ainda que o processo de trabalho do Nasf-AB exija práticas colaborativas e interprofissionais de apoio às EqSF, no desenvolvimento de ações de prevenção e promoção da saúde11. Esse fato contribui para os desafios na construção de competências e habilidades do fisioterapeuta à reorientação do modelo assistencial proposto na política da $\mathrm{APS}^{2,19}$.

Ainda que, neste estudo, as ações de EPS tenham sido raras, evidências ressaltaram a importância da EPS para fisioterapeutas atuantes no Nasf-AB, considerando a necessidade do trabalho em equipe, ao mesmo tempo que reconhece o desafio de concretizar essas ações para imprimir uma reorientação necessária das práticas de saúde centradas na APS $^{6-9,20}$.

As desigualdades de cobertura de APS entre os municípios da região pesquisada coadunam com o debate sobre as RM no Brasil, destacando como desafios a fragilidade institucional e de gestão dos sistemas de saúde. Reiteram-se, ainda, fenômenos de metropolização e regionalização sobrepostos, com tensões de natureza interinstitucional, dada a falta de estímulos para cooperações em pactuações regionais de saúde diante da ausência de fontes definidas e estáveis de financiamento ${ }^{21}$.
Os problemas de infraestrutura identificados neste estudo se assemelharam a outros achados da literatura que ratificam fragilidades estruturais dos sistemas municipais de saúde como limitadoras do processo de trabalho da fisioterapia na APS, sobretudo em municípios mais desiguais do Nordeste ${ }^{11,12,22}$.

A demanda por atendimentos individuais de fisioterapia na APS, em detrimento de ações coletivas, é uma problemática recorrente na relação fisioterapeuta-comunidade, também evidenciada em outros estudos que identificaram resistência da população às ações promotoras de saúde, propostas por fisioterapeutas, e a hipervalorização das ações curativas e reabtilitadoras ${ }^{23,24}$.

Neste estudo, merecem destaque os conflitos de atribuições entre fisioterapeutas e demais profissionais das equipes de APS. Em outros municípios brasileiros, grande parte dos conflitos entre fisioterapeutas e profissionais do Nasf-AB ocorria por diferença de carga horária de trabalho (os primeiros com 30 horas e os demais com 40 horas $)^{5,6,8}$, ou por sobrecarga de trabalho com demanda reprimida pelo serviço de fisioterapia ${ }^{9,11}$, por divisão da equipe por questões salariais ${ }^{12,13}$ e por fragmentação do processo de trabalho ${ }^{25}$.

Na região estudada, vale reiterar que a boa adesão dos demais profissionais da APS, no modelo Saúde da Família, às ações propostas por fisioterapeutas diferencia-se de outros municípios brasileiros, nos quais foi evidenciada grande resistência de profissionais, independentemente da modalidade de equipe ${ }^{24,25}$.

A desigualdade na relação de EqSF implantadas e do número de Nasf-AB para ofertar retaguarda foi realidade diante deste estudo, bem como de outros cenários nacionais, resultando, também, na priorização dos atendimentos individuais em detrimento da prevenção e da promoção da saúde ${ }^{6-9,11,25,26}$.

As dificuldades de comunicação dos fisioterapeutas com secretários de saúde e coordenadores de APS, evidentes neste estudo, sinalizam fatores restritivos 
semelhantes e identificados em outro município brasileiro, no qual, também, havia fragmentação da comunicação e burocracia da gestão para o atendimento das demandas dos profissionais de fisioterapia ${ }^{12}$.

A alta demanda de atendimentos de reabilitação nos municípios estudados revela a urgência de planejamentos e programações locais de saúde para monitoramento regular das necessidades e ações de reabilitação comunitária, além da necessidade de maior transparência sobre a dinâmica do processo de matriciamento para a população e EqSF, visando reorientar a compreensão equivocada desses atores sobre as atribuições do fisioterapeuta no Nasf-AB. Também, mostrou-se como perspectiva o aumento da cobertura de fisioterapeutas na APS. Assim como neste estudo, outras pesquisas destacaram a necessidade de novas contratações de fisioterapeutas para a APS $^{5-7}$ e, também, sua inserção mais frequente na construção de planejamentos e programações locais de ações desenvolvidos pelo Nasf-AB ${ }^{8,9}$.

Com relação aos desafios, as atribuições dos fisioterapeutas no Nasf-AB ainda são genéricas, e persiste a concepção restrita, tanto dos profissionais quanto da comunidade, sobre a fisioterapia como núcleo profissional centrado na doença. Além disso, mesmo com as proposições das Diretrizes Curriculares Nacionais para os Cursos de Fisioterapia, a reorientação da formação acadêmica para romper com o modelo biomédico se configura como um dos principais desafios apontados nesse e em outros contextos brasileiros ${ }^{9,12}$

\section{CONCLUSÕES}

Apesar da boa comunicação com a comunidade e equipes multiprofissionais, os resultados descritos nesta pesquisa apontaram importantes dificultadores no processo de trabalho do fisioterapeuta nos Nasf-AB, na RM de Salvador, Bahia, como: formação acadêmica; educação permanente; insuficiência na infraestrutura; baixa compreensão sobre as potencialidades da fisioterapia no Nasf-AB; dificuldades logísticas.

Espera-se que esses achados auxiliem na formulação de estratégias de gestão para uma atuação mais qualificada do fisioterapeuta nos Nasf-AB. Além disso, destaca-se a relevância de investigações futuras para análise da cobertura de profissionais de fisioterapia na APS, das condições de trabalho, dos vínculos profissionais, das ferramentas de trabalho e das experiências exitosas no âmbito local.

\section{REFERÊNCIAS}

1. Aquino R, Medina MG, Nunes CA, Sousa MF. A Estratégia Saúde da Família e o reordenamento do sistema de serviços de saúde. In: Almeida-Filho N, Paim JS. Saúde coletiva: teoria e prática. Rio de Janeiro: MedBook; 2014.

2. Brasil. Ministério da Saúde. Gabinete do Ministro. Portaria n ${ }^{\circ} 2.436$, de 21 de setembro de 2017. Aprova a Política Nacional de Atenção Básica, estabelecendo a revisão de diretrizes para a organização da Atenção Básica, no âmbito do Sistema Único de Saúde (SUS). Diário Oficial da União. 2017; 22 set.

3. Fausto MCR, Almeida PF, Bousquat A. Organização da Atenção Primária à Saúde no Brasil e os desafios para a integração em redes de atenção. In: Mendonça MHM, Matta GC, Gondim R, Giovanella L. Atenção primária à saúde no Brasil: conceitos, práticas e pesquisa. Rio de Janeiro: Fiocruz; 2018. p. 51-72.

4. Brasil. Ministério da Saúde. Núcleo de Apoio à Saúde da Família: ferramentas para a gestão e para o trabalho cotidiano. Série cadernos de Atenção Básica. Brasília: DF, Ministério da Saúde; 2014.

5. Braghini CC, Ferretti F, Ferraz, L. Atuação do fisioterapeuta no NASF: percepção dos coordenadores e da equipe. Fisioter 
Mov. 2016;29(4):767-776.

6. Bim CR, Gonzáles A. Distribuição territorial de fisioterapeutas no Paraná e inserção em equipes na atenção básica. Saude e pesqui (Impr.). 2020;13(1):83-91.

7. Fernandes SCS, Ros MA. Desconstruir para transformar: o perfil do fisioterapeuta para o Núcleo de Apoio à Saúde da Família. Fisioter Bras. 2018;19(2):249-258.

8. Braghini CC, Ferretti F, Ferraz L. Atuação do fisioterapeuta no contexto dos núcleos de apoio a saúde da família. Fisioter Mov. 2017;30(4):703-713.

9. Souza MO, Santos KOB. Atuação do fisioterapeuta no Núcleo de Apoio à Saúde da Família. Fisioter Mov. 2017;30(2):237-246.

10. Brasil. Ministério da Saúde. Portaria $n^{\circ}$ 2.488, de 21 de outubro de 2011. Aprova a Política Nacional de Atenção Básica, estabelecendo a revisão de diretrizes e normas para a organização da Atenção Básica, para a Estratégia Saúde da Família (ESF) e o Programa de Agentes Comunitários de Saúde (PACS). Diário Oficial da União. 2011; 24 out

11. Fernandes JM, Rios TA, Sanches VS, Santos MLM. As ferramentas do NASF nas práticas em saúde de fisioterapeutas. Fisioter Mov. 2016;29(4):741-750.

12. Braghini CC, Ferretti F, Ferraz L. Atuação do fisioterapeuta no NASF: percepção dos coordenadores e da equipe. Fisioter Mov. 2016;29(4):767-776.

13. Nascimento AAP, Inácio WS. Atuação fisioterapêutica no núcleo de apoio à saúde da família: uma revisão sistemática J Health Sci Ins. 2015;33(3):280-286.

14. Luna MMA, Lucena PL, Farias AEM, Melo VFC. O acompanhamento fisioterapêutico de crianças com sequelas de paralisia cerebral atendidas no NASF do município de Alagoa Nova. Rev Pesqui Cuid Fundam. 2018;10(3):70-73.

15. Moro M, Vieira KM. Dilemas na construção de escalas tipo Likert: o número de itens e a disposição influenciam nos resultados? Revista Gestão Organizacional. 2016;6(3):1-14.

16. Biscarde DGS. Gestão regional do SUS nas regiões metropolitanas de Salvador e de Fortaleza: instâncias, processos de pactuação e relações de poder [tese]. Salvador (BA): Universidade Federal da Bahia, Salvador; 2016.

17. Bahia. Caderno de Monitoramento e Avaliação da Atenção Básica (CAMAB) [Internet]. Salvador: Sesab; 2019 [acessado 2019 dez 5]. Disponível em: http:// www.saude.ba.gov.br/atencao-a-saude/ dab/camab/

18. Brasil. Ministério da Educação. Conselho Nacional de Educação. Câmara de Educação Superior. Resolução CNE/CES 4, de 19 de fevereiro de 2002. Institui Diretrizes Curriculares Nacionais do Curso de Graduação em Fisioterapia. Diário Oficial da União [Internet]. 2002 [acessado 2014 jul 7]; 4 mar. Disponível em: http://portal. mec.gov.br/cne/arquivos/pdf/CES042002. pdf

19. Miranda EFS, Amado CF, Ferreira, TPS. Percepção de gestores acerca da atuação e inserção de terapeutas ocupacionais na atenção básica à saúde. Cad Bras Ter Ocup. 2019;27(3):522-533.

20. Bandeira D, Damaceno AN, Weiller, TH, Lopes LFD. Avaliação da coordenação da atenção por usuários dos serviços de atenção primária à saúde. REME Rev Min Enferm. 2020;24:e-1278.

21. Lara NC, Mendes A. A regionalização e 
o financiamento do SUS na Região Metropolitana da Baixada Santista do Estado de São Paulo. Rev Fac Ciênc Méd Sorocaba. Lima et al 2015;17(1):37-42.

22. Borges KP. Competências para formação do fisioterapeuta no âmbito das diretrizes curriculares e promoção da saúde. Saude e Pesqui (Impr.). 2018;11(2):347-358.

23. Brondani SC, Rodrigues LS, Quatrin LB. Percepção de acadêmicos de fisioterapia sobre a promoção da saúde. Rev APS. 2018;21(1):86-92.

24. Souza, CG, Furlanetto TS, Rosa BN, Candotti CT. Papel do fisioterapeuta e outros profissionais da saúde nas ações de promoção da saúde no ambiente escolar. Rev. Baiana Saúde Pública. 2017;40(1):2318-2660.

25. Guedes MBOG, Lima F, Bartolomeu F, Pinheiro HV, Lopes JM, Souza CG. Atenção fisioterapêutica em duas unidades básicas de saúde em um município do Rio Grande do Norte: um contato humanizado. Rev APS. 2016;19(1):150-153. 